\title{
GPPS-CH-2020-0160
}

\section{Design and Optimization of a Nacelle for a UHBR Turbofan engine using an Intuitive Class Shape Transformation based parameterization}

\author{
Legin Benjamin, Constance Heykena, Jens Friedrichs \\ Institute of Jet Propulsion and Turbomachinery, \\ Technische Universität Braunschweig \\ I.benjamin@ifas.tu-braunschweig.de \\ Braunschweig, Germany
}

\author{
Carlos Márquez \\ Rolls-Royce Deutschland Ltd \& Co KG \\ Carlos.Marquez@rolls-royce.com \\ Dahlewitz, Germany
}

\begin{abstract}
This paper deals with the design of an isolated axisymmetric nacelle for an Ultra-High Bypass Ratio (UHBR) engine. The intake, fan cowl, bypass duct and bypass nozzle are designed and optimized using a nacelle design system, established by combining an automated CFD analysis framework with an optimization algorithm. The optimization algorithm is based on an adaptive response surface methodology (RSM), which uses Kriging approximation to generate the surface model. The entire nacelle is parameterized using an intuitive Class shape transformation (iCST) approach. The optimization was conducted separately for the intake-cowl and exhaust system assuming minimal aerodynamic interactions between the two. The final optimized design had 26.9 Drag counts at cruise. The response surface was able to predict the cruise drag to $6 \%$ accuracy. The optimized bypass nozzle gave a maximum velocity coefficient $\left(\mathrm{C}_{\mathrm{V}}\right)$ of 0.9914 at a corresponding discharge coefficient $\left(\mathrm{C}_{\mathrm{D}}\right)$ of 0.995 .
\end{abstract}

\section{INTRODUCTION}

Recent trends in civil aviation engines indicate an increasing bypass ratio (BPR) in order to improve fuel efficiency of the aircraft engine (Suder, 2013). A high BPR means a large fan diameter that would require a larger and heavier nacelle. Studies have shown a 30\% increase in nacelle drag for an increase in BPR from 7 to 14 (Daggett, 2002). The main functions of a civil engine nacelle are (i) To efficiently supply air to the fan with low distortions (ii) Expansion of gases through the exhaust system for optimum thrust performance. The first function is performed by the intake section of the nacelle and the second, by the ducts and nozzles of the bypass and core streams. Therefore, reducing the weight and drag for nacelles with high BPR without impacting the fan efficiency is one of the major challenges in nacelle design.

The class shape transformation (CST) approach (B.M. Kulfan, 2006) has demonstrated applicability to a wide range of aerodynamic shapes like the design of wing profiles, aircraft bodies and nacelles (Kulfan, 2008). It has proven to give superior performance compared to an ellipse or B spline approach, in regard to nacelle design and optimization (Albert, 2013). However, it has the drawback that the parameterization is not based on intuitive design variables. This was mitigated with the introduction of the intuitive CST approach (F. Zhu, 2013). The iCST method has been applied to design nacelle geometries with good aerodynamic performance using relatively few design parameters (Christie, 2016).

Existing literature contains several studies employing a CFD based nacelle aerodynamic optimization. Albert (Moritz Albert, 2014) constructed an automated 3D nacelle evaluation system that could obtain a pareto-optimal solution with the objective of minimizing the external peak Mach number at cruise and internal peak Mach number at crosswind condition. Robinson (Matthew Robinson, 2017) presented a multi-objective optimization study using a genetic evolutionary algorithm coupled with a response surface model, using cruise drag, drag rise Mach number and spillage drag between mid-cruise to end of cruise as objective functions. Heidebrecht (A. Heidebrecht, 2019) demonstrated the applicability of response surface methods in developing a surrogate model for preliminary nacelle design, which could predict nacelle cruise drag with 3.6\% accuracy and drag rise Mach number with a standard error of 0.014. Toubin (Toubin, 2014) also presented a multi-objective optimization based on an evolutionary algorithm that used the circumferential distortion index at crosswind and cruise drag as objective functions.

The second function of the nacelle is performed by the exhaust. A separate jet exhaust system is employed in this study, this means that a core-cowl separates the bypass and core streams. A consequence of high BPR is that a much 
higher mass flow rate is exhausted through the bypass exhaust nozzle compared to the core nozzle. Hence, the aerodynamic design of the bypass duct, nozzle and post-exit components would be more critical in view of overall engine performance and efficiency. The iCST method has been previously applied for the parameterization of the bypass duct and nozzle (Ioannis Goulos, 2016). The parameterization was later integrated into a CFD based optimization environment using RSM and genetic algorithms (Goulos, 2016). Using this approach a 3.4\% improvement in net propulsive force was observed. Other optimization studies in this domain have been performed for dual-stream plug nozzle (Heath, 2015), bypass duct to minimize total pressure losses (Clemen, 2012) and on bypass nozzle after body design (Ioannis Goulos, 2018).

The objective of this study is to establish a unified aerodynamic optimization system for UHBR engine nacelles, for both the intake-fan cowl and bypass exhaust systems. The application of the nacelle is a mid-range aircraft concept with UHBR engine having a BPR of 16. A detailed description of the underlying aircraft configuration and its specification can be found in (Sebatian Woehler, 2018). An iCST based parameterization is adopted for the intake-fan cowl and bypass duct and nozzle geometries. The optimization system consists of modules for parametric geometry creation, mesh generation and RANS flow solution. The optimization algorithm employed is based on an adaptive response surface methodology (Tosin, 2014). It has previously been applied for the optimization of turbine blades and multi-rotor mixed flow pumps (Stefano Tosin, 2014). The approach followed in this study is to perform single cruise point optimization for both intake-cowl and bypass systems. Separate off-design calculations would also be performed at crosswind to assess the practicality of the optimized configurations.

\section{METHODOLOGY}

The nacelle design and optimization process is split into two independent optimization steps: (1) Intake and fan cowl optimization (2) Bypass duct and nozzle optimization. The underlying assumption is that the aerodynamic interactions between the two components of the nacelle are minimal. In addition to this, the numerical boundary conditions for the engine inflow and outflow are decoupled with regard to the engine mass flow rate. The objective for the intake-fan cowl optimization is to have a minimum cruise drag, whereas for the bypass nozzle it is to maximize the net thrust of the engine stream tube.

\section{Optimization Algorithm}

An in-house optimization algorithm based on an adaptive response surface methodology was used for the presented study (Tosin, 2014). The work flow of the optimization algorithm is shown in Figure 1. Based on upper and lower bounds of the optimization parameters, a sample distribution is created using the Latin Hypercube Sampling (LHS). Each iteration of the optimizer performs a Design of Experiments (DoE) for an LHS design. The sample distribution is passed on to an automated CFD analysis system. It creates the geometry in CATIA, generates a hybrid mesh, performs the CFD computation using the DLR flow solver TAU (Dieter Schwamborn, 2006), and finally calculates the cost function values for the optimizer. Based on the DoE results from the CFD analysis, a mathematical model, the meta model (response surface), is generated using the approximate Kriging method, using the Matlab toolbox ooDACE (Ivo Couckuyt, 2014).Within the Meta model, a global minimum is searched for, using the trust region method, and computed again by the CFD analysis system. The convergence of the optimizer is based on how close the Meta model predicted value of the minimum is to the actual value from CFD analysis; that is how close the response surface is to the physical behaviour. If convergence is not met the process is repeated again after reducing the solution space in order to increase the convergence speed. A detailed description of the optimization algorithm can also be found in (Tosin, 2014).

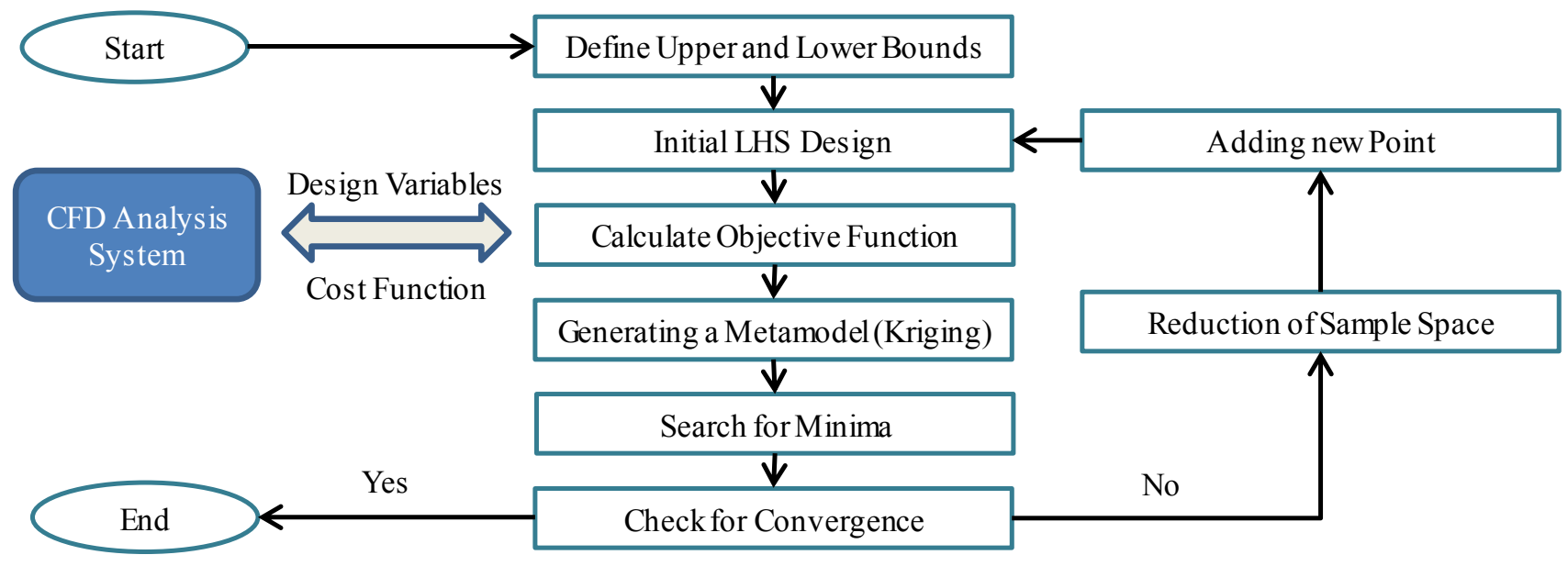

Figure 1 Work flow of optimization algorithm 


\section{Parameterization}

The mathematical approach described by Christie (Christie, 2016) was employed in this study for the parameterization of the intake and fan cowl curves. The basic theory is summarized below.

The geometry is represented as a product of a class function, $C$ and a shape function $S$,

$$
\varepsilon(\varphi)=\mathrm{C}(\varphi) \mathrm{S}(\varphi)+\varphi \Delta \varepsilon_{\mathrm{te}} ; \quad \varphi=\frac{\mathrm{x}}{\mathrm{c}}, \varepsilon=\frac{\mathrm{y}}{\mathrm{c}}
$$

The class function is of the form,

$$
\mathrm{C}_{N_{2}}^{N_{1}}=\varphi^{N_{1}}(1-\varphi)^{\mathrm{N}_{2}}
$$

The class function used for a round nosed airfoil is applied to the current nacelle profile, for which the exponents are $\mathrm{N}_{1}=0.5$ and $\mathrm{N}_{2}=1.0$. The shape function, which controls the shape of the final curve, is a product of Bernstein polynomials and weighting coefficients $\left(b p_{i}\right)$,

$$
\begin{aligned}
& \mathrm{S}(\varphi)=\sum_{i=0}^{N}\left[b p_{i} \cdot K_{i, n} \cdot\left(\varphi^{i} \cdot(1-\varphi)^{n-1}\right)\right] \\
& K_{i, n}=\frac{n !}{i !(n-i) !}
\end{aligned}
$$

The constraints on the geometry such as positional, local gradients and curvature are used to derive a system of equations where the only unknowns are the weighting coefficients of the Shape function. These take the form:

$$
\mathrm{A} \cdot \mathbf{X}=B
$$

By solving the system of equations for X, the CST curve using equation (1) is fully defined.

\begin{tabular}{|c|c|c|c|}
\hline Inner CST curve & & Outer CST c & \\
\hline R_LE & $\begin{array}{l}\text { Radius of curvature at the leading } \\
\text { edge of the nacelle }\end{array}$ & R_max/R_hi & $\begin{array}{l}\text { Ratio maximum cowl radius to the } \\
\text { radius at the highlight }\end{array}$ \\
\hline Intake length (L/D_fan) & $\begin{array}{l}\text { Length of the intake, computed as } \\
\text { a ratio to the fan diameter }\end{array}$ & f_max & Sets axial position of $\mathrm{R} \_\max$ \\
\hline Throat radius ( $\left.\mathbf{R}_{\text {throat }}\right)$ & Minimum radius of the intake & $\boldsymbol{\beta}$ & Boat tail angle of the nacelle \\
\hline Contraction ratio (CR) & $\begin{array}{l}\text { Ratio of throat area to the area at } \\
\text { the highlight }\end{array}$ & & \\
\hline Aspect ratio (AR) & $\begin{array}{l}\text { Controls the axial position of the } \\
\text { throat }\end{array}$ & & \\
\hline
\end{tabular}

\section{Intake and Fan Cowl}

The nacelle intake and fan cowl are formed by two $4^{\text {th }}$ order CST curves, meeting at the leading edge of the nacelle. The parameters used for defining the geometry are described in Table 1 and marked in Figure 2(a).

Table 1 Intuitive design parameters for Intake and fan cowl

\section{Bypass Duct \& Nozzle}

The iCST approach is extended for the parameterization of the bypass duct and nozzle. The parameterization

\begin{tabular}{|c|c|c|c|}
\hline \multicolumn{2}{|l|}{ Outer curve } & \multicolumn{2}{|l|}{ Inner curve } \\
\hline Duct length (1_duct) & Total length of duct \& nozzle & Nozzle length & $\begin{array}{l}\text { Defines the axial distance between } \\
\text { nozzle exit and charging planes }\end{array}$ \\
\hline $\begin{array}{l}\text { Nozzle Area ratio } \\
(\text { A_cp/A_exit) }\end{array}$ & $\begin{array}{l}\text { Ratio of area at the charging plane } \\
\text { to that at the nozzle exit }\end{array}$ & R_max_nozzle & $\begin{array}{l}\text { maximum radius of the inner curve } \\
\text { at the charging plane }\end{array}$ \\
\hline Slope at $\mathrm{CP}\left(\boldsymbol{\theta} \_\mathrm{CP}\right)$ & $\begin{array}{l}\text { Sets the slope of the curve at the } \\
\text { charging plane }\end{array}$ & B_core cowl & $\begin{array}{l}\text { Dependent parameter that defines } \\
\text { the slope of the nozzle after-body }\end{array}$ \\
\hline B_outer_duct & Controls the slope at nozzle exit & & \\
\hline
\end{tabular}
implemented is for a convergent bypass nozzle (and duct),shown in Figure 2 (b) and described in table 2; this parameterization is derived from that presented by Goulos (Ioannis Goulos, 2016). The inner and outer curves of the bypass nozzle are each described by two CST curves; the nozzle after-body is formed by a straight line.

\section{Table 2 Intuitive design parameters for bypass duct \& nozzle}




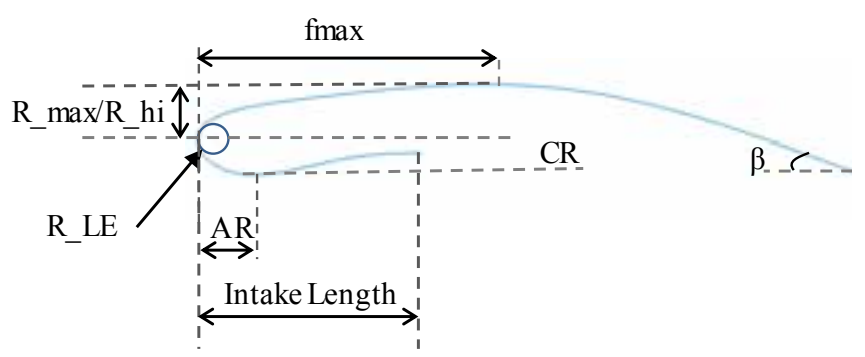

(a) Intake and Cowling

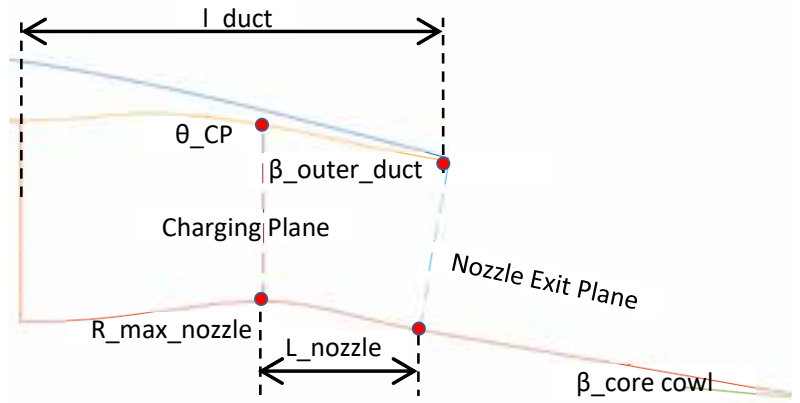

(b) Bypass duct \& Nozzle

Figure 2 iCST Parameterization

\section{Numerical Setup}

Steady state compressible RANS simulations were performed using the DLR flow solver TAU, which is an unstructured (hybrid grids) $2^{\text {nd }}$ order finite volume based CFD solver. A $2^{\text {nd }}$ order central scheme with scalar dissipation is used for the spatial discretization of convective fluxes and the viscous fluxes are discretized with central differences. Time integration is performed using a backward Euler implicit scheme, with a CFL number of 1.8 used for the fine grid level. Local time-stepping and a ' $3 \mathrm{v}$ ' multigrid cycle approach are employed to accelerate convergence. The convergence criteria is that all normalized density and momentum residuals should be of the order of $10^{-6}$.

The aim of the optimization was to design an axisymmetric nacelle, hence only a sector of $1^{\circ}$ was simulated using axisymmetric boundary condition. Cruise conditions at an altitude of $37,000 \mathrm{ft}$ are used for the optimization studies, so reference pressure and temperature were set correspondingly. Cruise Mach number was 0.83 . The domain size was 30 times the fan diameter. A far field boundary condition was used at the outer domain boundaries. The engine intake is modelled using a mass flow rate boundary condition; the mass flow boundary condition was dictated by a constraint from the fan design model. The exhaust boundaries (bypass and core) are specified by the total pressure and total temperature at these stations corresponding to the cruise operating point of the engine. It should be noted that the intake and exhaust boundary conditions are decoupled from each other. The Spalart Allmaras (SA) model was used for modelling turbulence. All walls of the nacelle were treated as fully turbulent and no transition modelling was applied. Hybrid meshes were generated using the meshing package Centaur. The mesh size was approximately 400,000 elements; this was the result of a grid convergence study, which is addressed in the following section. The first cell was sized to have a $\mathrm{Y}+$ of close to 1 .

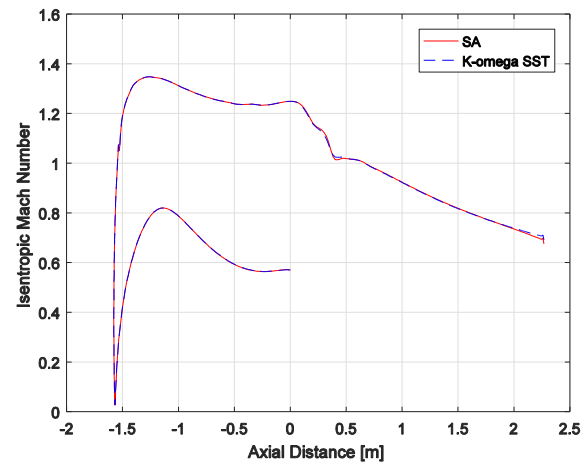

Figure 3 : Comparison of SA and SST models at cruise

For the crosswind test cases, full annulus simulations were performed on a nacelle half model (without the exhaust) at sea level conditions with a crosswind speed of 30knots/s, the fan face was modelled as a pressure outlet to give an intake mass flow rate close to take-off conditions. K-omega SST was used for turbulence modelling as it has a better separation prediction compared to the SA model. The mesh was sized again to have a $\mathrm{Y}+$ of close to 1.

Figure 3 shows the isentropic Mach number distribution for a design candidate at cruise condition for both SA and K-omega SST turbulence models. The curves are coincident with each other, indicating that both models predict similar aerodynamic behaviour for the nacelle configuration investigated at cruise. Since the SA model has the advantage of quicker solution time, as it solves for only one turbulent transport equation when compared to SST model which solves 
for two turbulent transport equations, the SA model is chosen for investigations in cruise condition. The SA model is also known to under predict separation, hence the choice of K-omega SST for off-design calculations.

\section{Meshing strategy and Grid independence study}

A grid study was performed to assess the grid sensitivities and select the mesh for the optimization study. A surface mesh was generated using the meshing package from Centaur, which was then later extruded circumferentially by $1^{\circ}$ with a unit cell width using the pre-processing capabilities of TAU. A hybrid meshing approach is followed with structured hexahedral elements (quads in 2D) in the near wall regions for boundary layer resolution and tetrahedral (triangular elements in 2D) for the rest of domain. Sources were defined to increase the mesh refinement in regions of interest such as near field of the nacelle, intake, bypass and core exhausts. In figure 4(a) the refinement in the intake and leading edge of the nacelle lip can be seen. These sources are sized such that all designs generated during the optimization study are within the tolerance regions of the sources, ensuring adequate quality for all meshes generated.

Five meshes of increasing refinement were created containing $4.7 \times 10^{4}, 1.01 \times 10^{5}, 1.73 \times 10^{5}, 4.41 \times 10^{5}$ and 8.29 $\mathrm{x} 10^{5}$ elements respectively. The $\mathrm{Y}+$ for all the meshes were close to 1 . The local and global element sizes were decreased by factor of approximately 1.3-1.4 for each progressive mesh, the growth ratio for the structured hexahedral region was also reduced from a factor of 2.0 for the coarsest mesh to 1.05 for the finest mesh to get a closer packing of the cells, whilst increasing the number of layers to maintain a constant thickness of the structured region for all meshes generated. Each mesh was simulated at the cruise condition, with boundary conditions as described in the earlier section.

Figure 4(b) shows the results of the grid study for the parameter intake pressure recovery (IPR). IPR exhibits a monotonic convergence characteristic for all meshes investigated and approaches an asymptotic region above $4 \times 10^{5}$ elements. The grids with $1.73 \times 10^{5}, 4.41 \times 10^{5}$ and $8.29 \times 10^{5}$ elements were used to evaluate the grid convergence index as per the approach described by (Celik, 2008). The GCI values obtained were $0.00226 \%$ for the mesh with $4.41 \mathrm{x}$ $10^{5}$ elements and $0.00051 \%$ for the finest mesh. Based on the grid convergence characteristic shown in Figure 4(b) and the GCI values, the $4.41 \times 10^{5}$ element mesh selected for the optimization study. The intake portion of this mesh is displayed in figure 4(a), the resulting triangular elements within the intake are $15 \mathrm{~mm}$ in edge length.

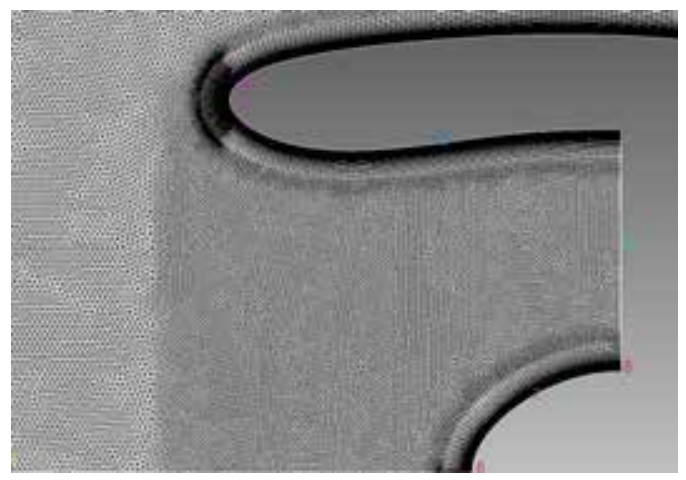

(a) view of selected mesh

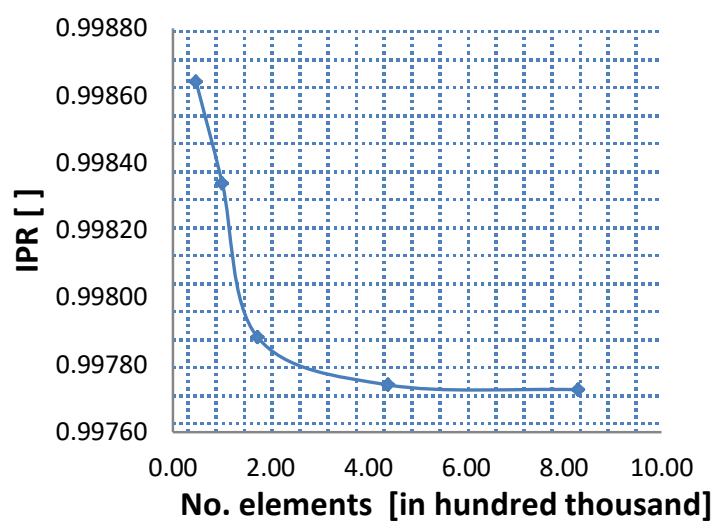

(b) IPR- No. of elements

Figure 4 : Grid independence results

\section{Optimization strategy - Decoupled Intake and exhaust systems}

The intake and exhaust boundary conditions, described in the earlier section, are decoupled from each other. One reason for such an approach is to investigate the performance of the intake and exhaust system solely on the basis of engine cycle constraints; which are the mass flow rate constraint for the fan and the nozzle pressure ratios for the bypass and core nozzles, without taking any interaction effects into consideration. The decoupled approach is also supported by the results of (Goulos, 2016) who concluded from his study on bypass nozzle optimization that the design changes applied to the exhaust system did not influence the performance of the intake or that of the nacelle forebody. The advantage of the approach from an optimization point of view is that the intake and bypass optimization loops could be run independently and in parallel to each other. This means the nacelle intake and cowl optimization run was performed with a non-optimized bypass geometry which remained unchanged for the entire optimization process. Similarly, the bypass optimization run was performed with a non-optimized intake. In the end these two designs merged together to 
obtain the final optimized design. A discussion on the consequences of this assumption and the adopted workflow is provided towards the end of the paper.

\section{A) INTAKE AND FAN COWL OPTIMIZATION}

The objective of the intake and cowl optimization is to design a nacelle with minimum drag and good pressure recovery at cruise conditions. The objective function for the optimizer is the total drag of the nacelle profile. This includes the external cowl drag plus the drag of the pre-entry engine stream tube.

Two optimization loops are presented, the design parameter ranges are listed in Table 3. The objective for loop1 was to perform a design space exploration. Hence, both intake and fan cowl parameters were varied. Loop2 was designed as an improvement over loop1. The parameter ranges for loop2 were adapted on the basis of the off-design behaviour seen for the loop1 optimum design, which will be addressed later. Most importantly, CR was increased to a value of 1.34 , and the parameter controlling the radius of curvature at the leading edge was increased by $50 \%$. Starting with an initial population size 200, loop1 evaluated close to 1000 designs over 10 iterations of the optimizer, whereas loop 2 evaluated about 800 designs over 14 iterations. The optimum parameter set for both loops are also listed in Table 3 . Loop1 results will be presented for the discussion of intake parameter trends, as most intake parameters were held constant for loop2. Both loops showed similar trends for the external parameters, so only loop2 results will be presented.

\section{Table 3 Intake-fan cowl optimization summary}

\begin{tabular}{ccccc}
\hline Parameter & Range loop1 & Optimum set & Range loop2 & Optimum set \\
\hline $\mathrm{R}_{\text {throat }} /$ R_fan & $0.93-0.999$ & 0.9318 & 0.9391 & 0.9391 \\
CR & $1.15-1.30$ & 1.24 & 1.34 & 1.34 \\
AR & $1.75-3.50$ & 2.2 & $1.75-3.00$ & 2.4 \\
Intake L/D & $0.40-0.55$ & 0.49 & $0.50-0.65$ & 0.56 \\
R_LE & $0.05-0.10$ & 0.05 & 0.075 & 0.075 \\
R_max/R_hi & $1.10-1.15$ & 1.15 & $1.12-1.25$ & 1.175 \\
f_max & $0.2-0.45$ & 0.33 & $0.25-0.5$ & 0.35 \\
$\beta[$ [deg] & $8-20$ & 10 & 15 & 15 \\
\hline
\end{tabular}

\section{Intake Parameter Trends}

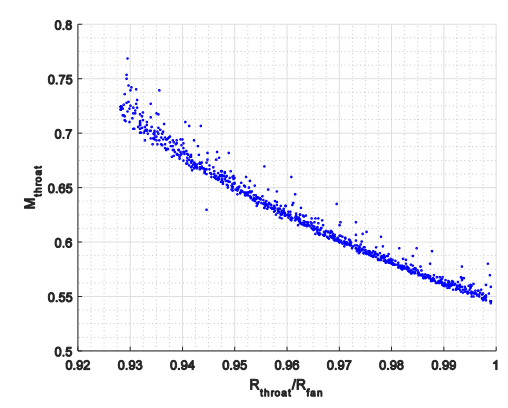

(a) $M_{\text {throat }}-R_{\text {throat }} / R_{-}$fan

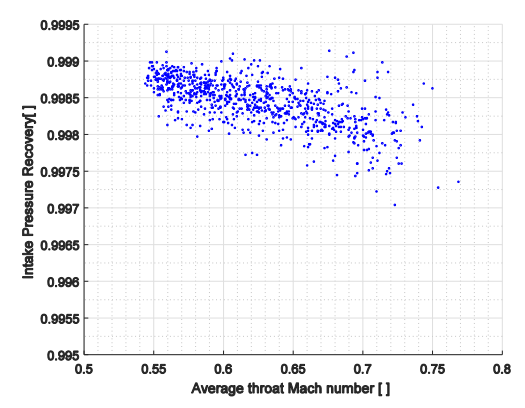

(b) $M_{\text {throat }}$ - IPR

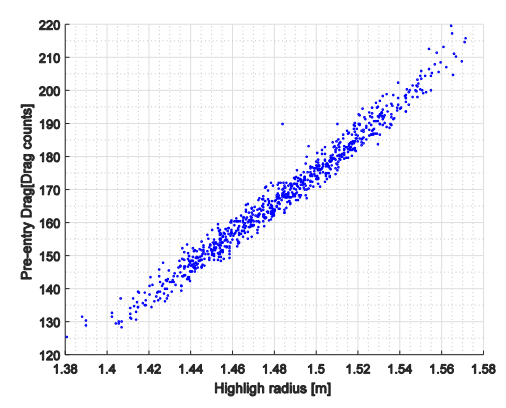

(c) R_hi - Pre-entry drag

Figure 5 Trends for intake

Throat radius: The most significant intake performance parameter was found to be the throat radius. Figure 5(a) shows the throat radius (non-dimensionalized) plotted against the averaged throat Mach number. The throat Mach number bears an almost linear inverse proportional relation to the throat radius. Figure 5(b) shows the relation between the intake pressure recovery (IPR), which is the ratio of total pressure at the free stream to that at the fan face, and the average throat Mach number. A roughly inverse linear relation exists between the two parameters, but with a very low gradient. The IPR is within the range 0.9975-0.999, lower the throat Mach number (higher throat radius), higher the pressure recovery. It is worthwhile to note that there were no shocks within the intake for the range of designs evaluated; the presence of local shocks at the throat will drastically reduce the IPR. According to (Viall, 1968), this reduction in IPR occurs above an average throat Mach number of 0.8 .

Highlight radius: The highlight radius is a function of throat radius and contraction ratio. Figure 5(c) shows the relation between the highlight radius and pre-entry drag. A stark increase in pre-entry drag for increased highlight radius can be observed due to the rise in curvature of the pre-entry stream tube with increase in capture area for a given engine mass flow rate (mass flow capture ratio (MFCR) decreasing). A conflicting requirement becomes obvious here, for a lower 
pre-entry drag, a lower highlight radius and hence lower throat radius is required, whereas intake pressure recovery favours a higher throat radius.

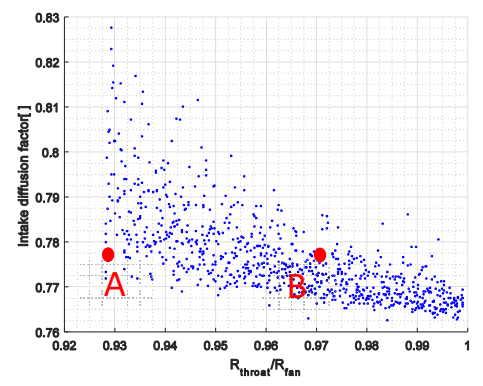

(a) Diffusion factor

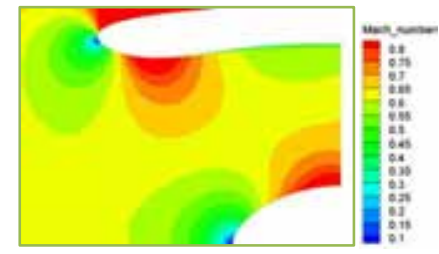

A

(b) Long Intake

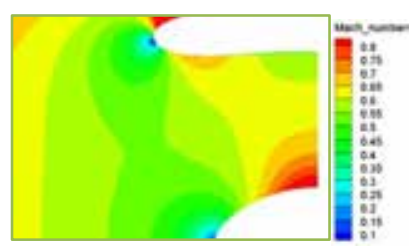

B

(c) Short Intake

Figure 6 Intake diffusion

Intake length: The intake diffusion factor is the ratio of the average Mach number at the Fan face ( $\mathrm{M}_{-}$fan) to the free stream Mach number. Figure 6(a) shows the relation between the Intake diffusion factor and the non-dimensionalized throat radius. Unlike the throat Mach number in Figure 5(a), the diffusion factor (M_fan) is not solely a function of the throat radius. The spread in the plot can be explained taking into consideration the effect of the intake length. Longer intake length means an increase in the length of the diffusor section of the intake i.e., from the throat to the nose of the spinner. Hence, the flow would undergo a greater deceleration for longer intakes. Note that the flow channel area increases from the throat to the nose of the spinner; there on it decreases till the fan face. Therefore, a long intake with smaller throat radius can have the same diffusion effect as a shorter intake with larger throat radius. This effect is demonstrated in the two contours in Figure 6(b) and (c).

\section{Fan Cowl Parameter Trends}

The parameters that influence the external nacelle drag are discussed in this section. The two important factors that influence the external drag are the skin friction of the wetted surfaces and the shock strength. The target of the optimization study was to find the right balance between these two factors. The nacelle drag presented here is expressed in terms of drag counts ( $1 \mathrm{drag}$ count $=1 \times 10^{-4}$ of the drag coefficient), using the aircraft wing area as reference.

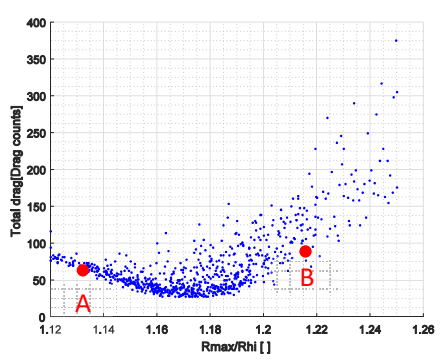

(a) Rmax

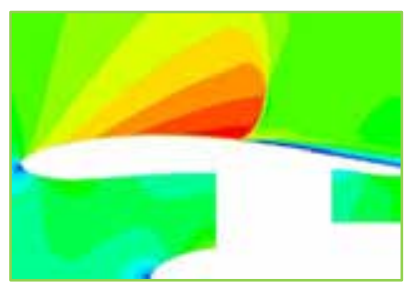

B

(d) Rmax

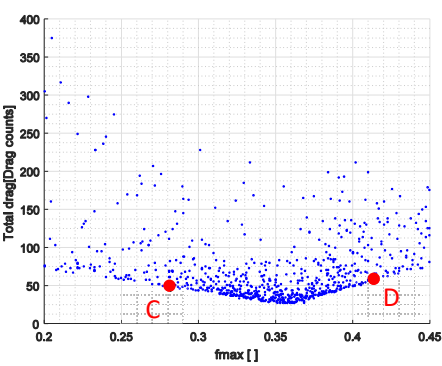

(b) f_max

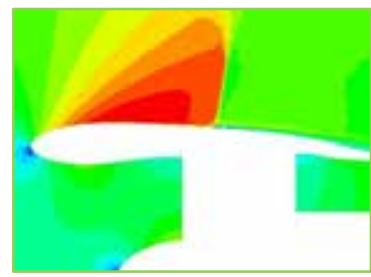

C

(e) f_max

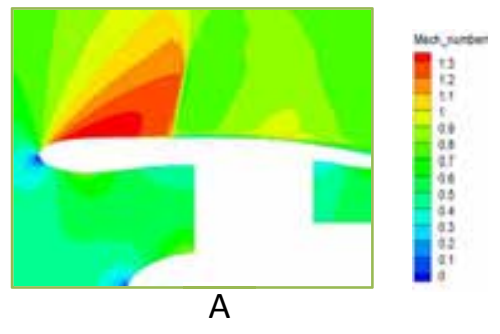

(c)Rmax

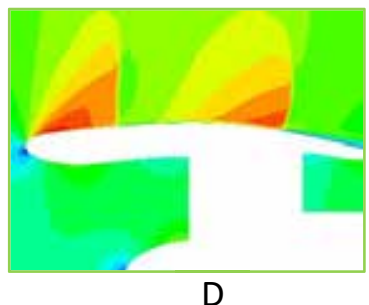

(f) f_max

Figure 7 Fan cowl parameters

Maximum Nacelle Diameter: The total drag and $\mathrm{R}_{-}$max/R_hi ratio is plotted in Figure 7(a). A bowl shaped distribution is formed, with the minima occurring at an $R_{-} \max$ ratio of 1.175 . The shape of the distribution can be interpreted in terms of the shock strength; the left portions of the distribution are formed by designs that are more flat, as in design 
A(Figure 7 (c)), this results in a high curvature near leading edge which causes a strong shock to form near the front of the nacelle. The right portion of the distribution is formed by designs that have a very large external diameter, design $\mathrm{B}$ (Figure $7(\mathrm{~d})$ ); this also results in a strong shock over the maximum diameter position.

Position of Maximum Diameter: The parameter f_max which controls the axial location of the maximum diameter also forms a bowl shaped distribution with the resulting drag, as shown in Figure 7(b). The minimum is formed close to 0.35 . For the distributions to the left of the minimum, like design $\mathrm{C}$ (Figure 7 (e)), it can be seen that the suction peak is located aft of the maximum diameter position, hence the lip suction force is directed in the drag direction (towards the right- in the direction of flow) as opposed to the conventional thrust direction, this causes a reduction/absence of the lip suction thrust which leads to an increase in drag as the maximum diameter is shifted to the front. This is also coupled with a strong normal shock, which further increases the drag. For higher values of $\mathrm{f}$-max, the maximum diameter is shifted further downstream, design D (Figure 7(f)), and causes a second shock downstream of the R_max position leading to an increased drag.

\section{Performance of Optimum design candidates}

The optimum design parameters for loop1 and loop 2 are listed in Table 3. Their shape can be inferred from the respective contour images shown in Figure 8. The key performance metrics are shown in Table 4. Loop2 design results in 4 drag counts higher than the loop1 design at cruise. As a consequence of the increased highlight radius for the loop2 design (increased $\mathrm{R}_{\text {throat }}, \mathrm{CR}$ ) the mass flow capture ratio for loop 2 is much lower than loop 1 . This results in a higher pre-entry drag (Figure 5 (c)) which contributes to the increase in total cruise drag. Lower MFCR also results in a stronger acceleration around the lip of the nacelle leading to higher peak Mach number around the lip. This in turn, leads to a relatively stronger shock for the loop2 design, shown by the step in its Isentropic Mach distribution, Figure 8(c), this also contributes to the increased cruise drag. The IPR is slightly lower for the loop 2 design on account of increased viscous losses due to its increased length. The Isentropic Mach distribution for the intake portion reveals a peak Mach number of 0.9 for loop 1 and 0.8 for loop 2 near their respective throat positions; this indicates that loop2 would have a higher margin for an increase in intake mass flow before any internal shocks would occur.

Table 4 Performance metrics for optimized candidates

\begin{tabular}{lccccc}
\hline & MFCR & Cruise drag & IPR (cruise) & M_fan (cruise) & DC60 (crosswind) \\
\hline Loop1 & 0.75 & 23 & 0.9985 & 0.64 & -0.411 \\
Loop2 & 0.68 & 26.9 & 0.9978 & 0.64 & -0.015 \\
\hline
\end{tabular}
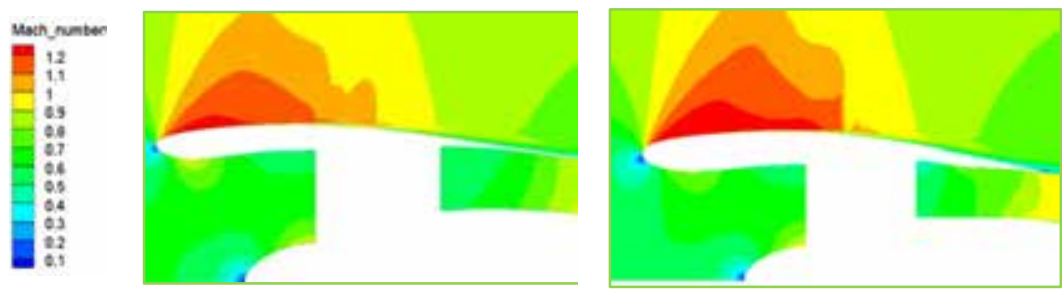

(a)Loop1 Optimum
(b)Loop2 Optimum

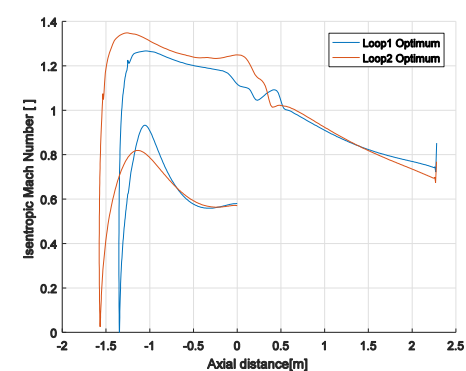

(c) Isentropic Mach comparison

Figure 8 Comparison of Optimized configurations

Figure 9 shows both designs operating at 30knots crosswind and 100\% take-off mass flow rate at sea level. Loop 1 intake is heavily separated right from the leading edge. The contour at the aerodynamic interface plane (AIP) shows a large distortion in the flow to the fan, which is reflected in its DC60 value (Pavlos K. Zachos, 2017) in Table 4. Loop2 design on the other hand shows a fully attached flow, this is a direct consequence of the increased CR and radius of curvature at the leading edge.

While loop 2 design is seen to be far superior to loop 1 design in off-design performance, the major drawback comes in terms of the higher cruise drag. The solution to this would be a non-axisymmetric three dimensional nacelle, which has a shape similar to loop2 design at the maximum half breadth (windward) sections and that similar to the loop1 design for the crown section (top) and somewhere in between the two for the keel section (bottom) to have good performance when the nacelle is operating at high incidence. 


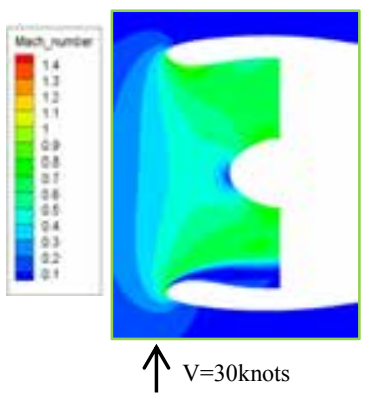

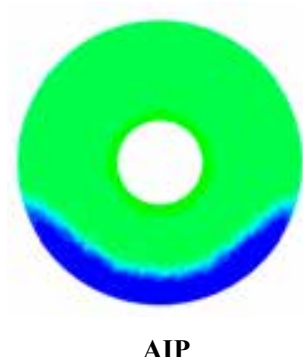

AIP
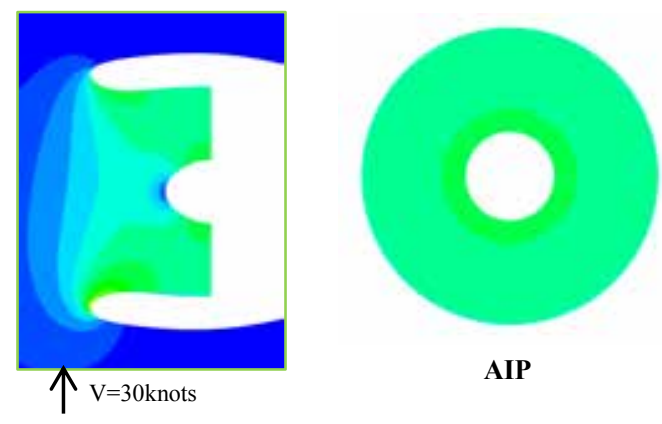

AIP

(a)Loop1 Optimum

(b)Loop2 Optimum

Figure 9 Comparison of crosswind performance

Loop2 optimum design was further investigated to evaluate its performance at high incidence. Figure 10(a) shows the plot of DC60 against the angle of attack for low speed conditions ( free stream Mach $=0.25$ ), Figure 10(b) shows the same at high speed conditions ( free stream Mach $=0.83$ ). At low speed conditions the intake operates without separation up to an angle of $34^{\circ}$, figure 10 (c) shows the intake operating at $34^{\circ}$ AoA. The lower lip (keel section) is the critical factor in this scenario, the stagnation point lies on the outer cowl for the lower lip, shocks are formed after the leading edge of the lower lip. Nevertheless, this does not cause a shock induced separation or an increased boundary layer thickening. At high speeds the intake is able to operate without separation up to an angle of about $12^{0}$. Both these results indicate that the intakes designed with the proposed design strategy are capable of a good high incidence performance.

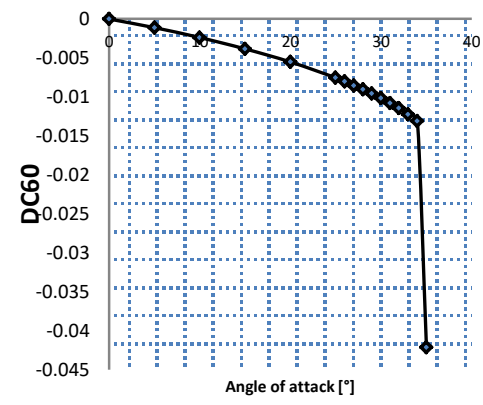

(a)high incidence (low speed)

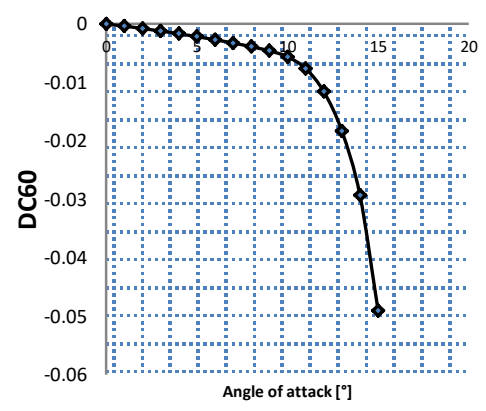

(b)high incidence (cruise)

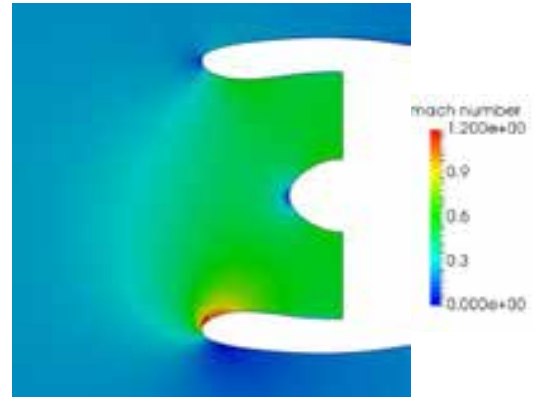

(c) Intake operating at high incidence

Figure 10 High Incidence performance of the intake

\section{Convergence of the Optimizer}

The convergence criteria is based on how close the value predicted by the meta model comes to the actual calculated results from CFD, that is how close the green curve comes to the blue curve in figure 11. For the first iteration, the difference between the two curves is greater than 50DC, it decreases for subsequent iterations and eventually after 14 iterations the difference is less the $1.6 \mathrm{DC}$, indicating convergence. This also means that the response surface can predict the cruise drag within an accuracy of $5.9 \%$, which is a slight improvement from the $8 \%$ error margin in cruise drag predicted by the published ESDU method (ESDU, 1981).

\section{B) BYPASS DUCT \& NOZZLE OPTIMIZATION}

The parameterization shown in Figure 2(b) was used for the bypass optimization. The objective of the optimization was to maximize the net thrust of the engine stream tube at cruise operating point. The thrust used here accounts for all losses (including viscous wall loss) from the bypass/core entry plane to far downstream within the engine stream tube (forces on the external fan cowl are not included). At cruise the bypass nozzle operates at a pressure ratio higher than the critical pressure ratio for air (1.89), hence the bypass nozzle is chocked at cruise. The parameter ranges used for the study 
and the resulting optimum are listed in Table 5. In total, about 900 designs were evaluated over 8 iterations of the optimizer.

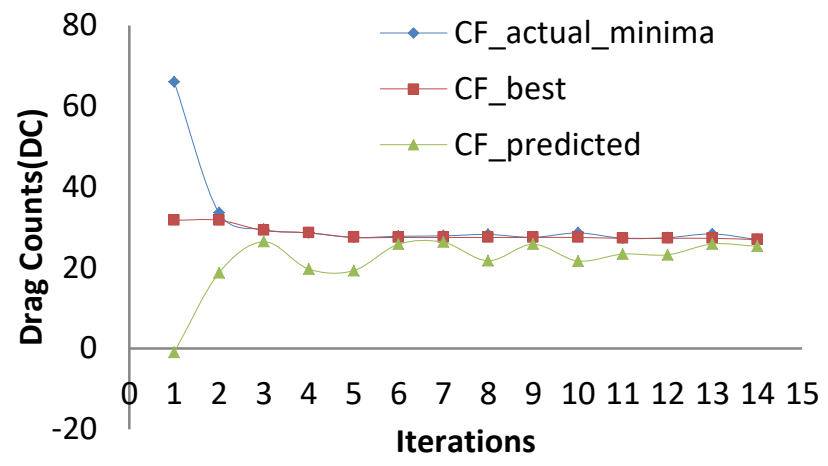

Figure 11 Optimizer Convergence

Table 5 Bypass nozzle optimization overview

\begin{tabular}{|c|c|c|}
\hline Outer curve & Range & Optimum \\
\hline I_duct/D_fan & $0.36-0.47$ & 0.437 \\
\hline Nozzle Area ratio (A_cp/A_exit) & $1.1-1.25$ & 1.1 \\
\hline @_CP $\left[{ }^{\circ}\right]$ & 3-7 & 3.069 \\
\hline B_outer_duct $\left[{ }^{\circ}\right]$ & $7-15$ & 7 \\
\hline \multicolumn{3}{|l|}{ Inner curve } \\
\hline Nozzle length: I_duct & $0.35-0.65$ & 0.565 \\
\hline R_max_nozzle (non-dimensionalized) & $1.0-1.1$ & 1.0 \\
\hline
\end{tabular}

\section{Parameter trends}

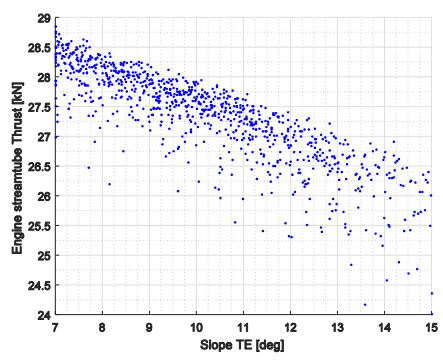

(a) B_outer_duct

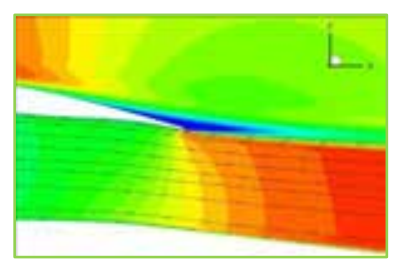

(d) B_outer_duct

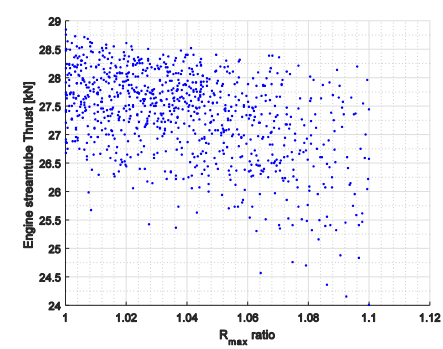

(b) R_max (inner curve)

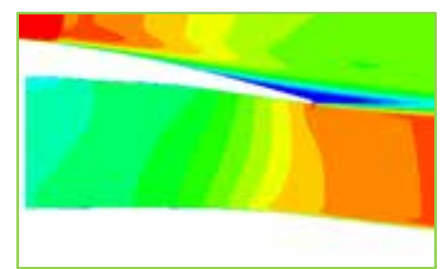

(e) R_max

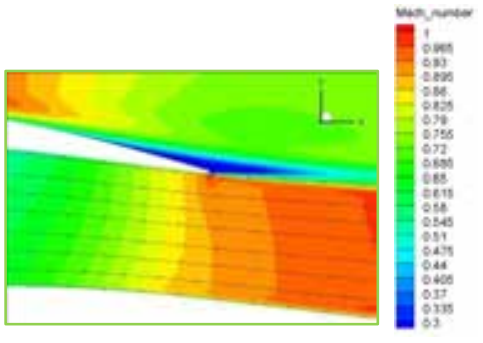

(c) B_outer_duct

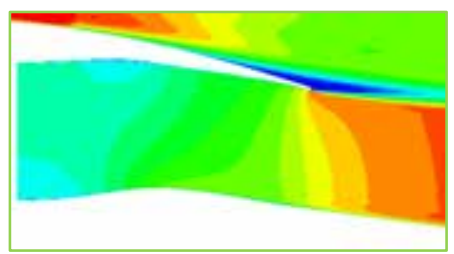

(f) R_max

Figure 12 Bypass nozzle parameter trends

Exit slope: The relation between the net engine stream tube thrust and the exit slope is shown in figure 12(a). It seems to bear a linear inverse proportional relation to the engine thrust with a high slope. The lower bound of the exit slope angle gave the best thrust behavior; the physical explanation for this can be deduced from the contour images shown in figure 12(c) and (d). For the design in figure 12(c), with a slope angle of $7^{\circ}$, the streamlines are more aligned to the inner and 
outer nozzle aero-lines compared to the design in figure $12(\mathrm{~d})$, with a slope angle of $13^{\circ}$. Furthermore, the Mach contour lines are more perpendicular to the nozzle aero-lines at the exit, for the design figure 12(c).

Maximum radius for inner curve: This parameter also bears an inverse relation to the thrust. The maximum radius influences the radial distribution of pressure (and Mach number) at the nozzle charging plane. The contour image in figure $12(\mathrm{e})$, is for a maximum radius ratio of 1.0 (flat design) and for figure 12(f) is for a ratio of close to 1.1 (with a more definitive hump). The radial distribution of Mach number at the charging plane is more uniform for the design in figure 12(e); this in turn leads to a more aligned flow at the nozzle exit, indicated by the perpendicularity of the Mach contours to the nozzle aero-lines.

\section{Optimized design and characteristics}

The flow field of the optimized nozzle is shown in figure 13(a). The design parameters for the nozzle are listed in Table 5. The resulting parameters are in line with the discussion of the previous section, exit slope, maximum radius ratio and nozzle area ratio converges to the lower bound, nozzle length converges closer to the upper bound. This indicates that for the current UHBR engine a more flat bypass duct and nozzle shaped such that the mean flow is aligned to the aero-lines at the nozzle exit, gives the best performance.

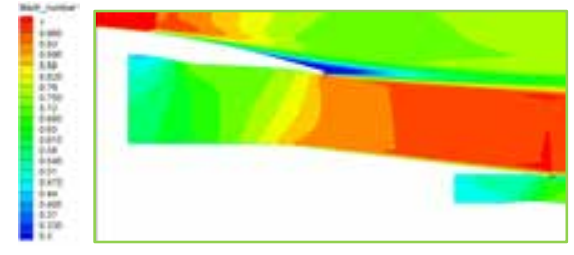

(a)Optimized flow-field

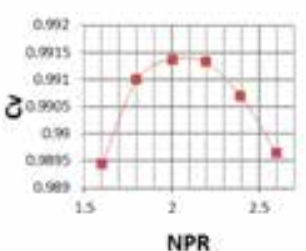

(b) $\mathrm{C}_{\mathrm{v}}$ characteristics

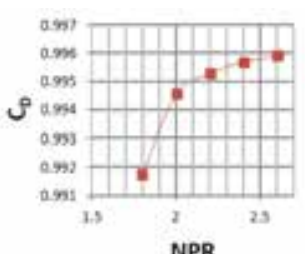

(c) $C_{D}$ characteristics

Figure 13 Optimized nozzle performance

The characteristics for the optimized bypass nozzle were derived at static sea level conditions. This is shown in figure 13(b) and (c). The shapes of the curves are typical for a convergent nozzle. The peak for the nozzle velocity coefficient $\left(\mathrm{C}_{\mathrm{V}}\right), 0.9914$, occurs close to a NPR of 2.0, which is close to the NPR at cruise, indicating that the nozzle would operate at maximum efficiency at cruise. The discharge coefficient $\left(\mathrm{C}_{\mathrm{D}}\right)$ curve increases monotonically up to 0.995 (at NPR 2.0) and then flattens there forth as the nozzle has chocked.

\section{Combining Intake and exhaust designs}

Here some consequences of the assumption of minimum aerodynamic interaction and the adopted workflow are discussed. The Mach contours for the optimized Loop1 intake (a), optimized nozzle(b) and the two combined (c) are shown in figure 14. Case (c) has a drag of 23.22Drag counts (DC) and case (a) has 23.004DC, the marginally higher drag count of $0.2 \mathrm{DC}$ can be attributed to the difference in lengths of the two nozzles (the length of the nozzle determines the aft length of the nacelle). However, this difference in drag is very marginal. Case (b) has a thrust coefficient (Thrust coefficent $=$ Net propulsive force $/ 1.4 * \mathrm{P}_{\text {ref }} * \mathrm{M}_{\text {ref }}{ }^{2} * \mathrm{~A}_{\text {ref }} / 2$ ) of 0.02418 and case (c) has a thrust coefficient of 0.02547. The lower thrust coefficient for case (b) can be attributed to the suppression effect of the bypass flow due to the external flow from the fan cowl. The static pressure at the trailing edge of the non-optimized cowl used in case (b) is much higher than that of the optimized cowl of case (c), which in turn leads to an increase in static pressure in the bypass jet post-exit stream tube, thereby decreasing the thrust force. A more in-depth analysis of the suppression effect can be found in (John J. Otter, 2018). Hence, the fan cowl pressure distribution has an influence on the nozzle performance at cruise and the assumption of minimal aerodynamic interaction between both systems is not exact. Nevertheless, from the above discussion it is clear that the adopted workflow ensured that this interaction (suppression of the bypass flow) was minimized.

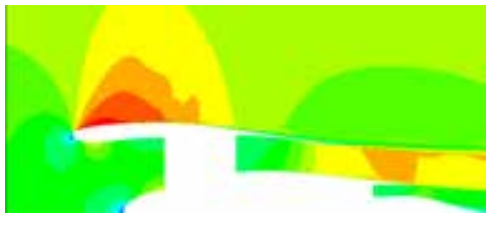

(a)Optimized Intake

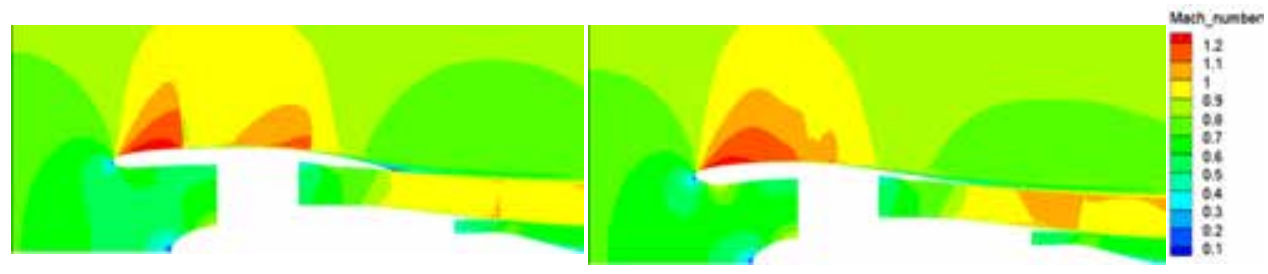

(b) Optimized nozzle (c) Optimized Intake+Nozzle

Figure 14: Combining Intake and nozzle optimized designs 


\section{OUTLOOK - 3D DESIGN ASPECTS \& INSTALLATION CONSIDERATIONS}

As mentioned in the introduction, the presented work is an initial phase in designing an over-wing-nacelle (OWN) (John R. Hooker, 2013) for the AVACON aircraft (Sebatian Woehler, 2018). In this regard, the presented approach has been extended for the design of 3 dimensional intakes, allowing for the local control on 8 sections of the nacelle as shown in figure 15 (a) \& (b) and the possibility to scarf the intake. Figure 15 (c) shows the Loop2 nacelle installed in an OWN configuration operating at cruise, the aircraft is at a trim angle of attack of $3.5^{\circ}$. There seems to be a flow straightening effect due to the wing (upstream of the nacelle) thereby, reducing local angle of attack of the nacelle to almost $0^{0}$. The corresponding DC60 value at the fan face is quite low, - 0.0098, this is further supported by the Mach contour at the fan face which is axisymmetric figure 15(d). Such a low distortion value owing to the OWN configuration further justifies the decoupling of the intake and bypass nozzle and the assumption of minimal aerodynamic interactions. Figure 15(f) shows the nozzle characteristics (Velocity coefficient) of the optimized nozzle with pylon, the reduction in the peak is about $0.07 \%$ due to the losses incurred by the pylon surfaces. Figure 15(e) shows the flow field for the nozzle with pylon operating under static conditions at an NPR of 2.0, shark fin type geometry is used for the external pylon model. The small reduction in peak of the $\mathrm{C}_{\mathrm{v}}$ characteristics demonstrates the applicability of the approach in designing practical bypass nozzle geometries.

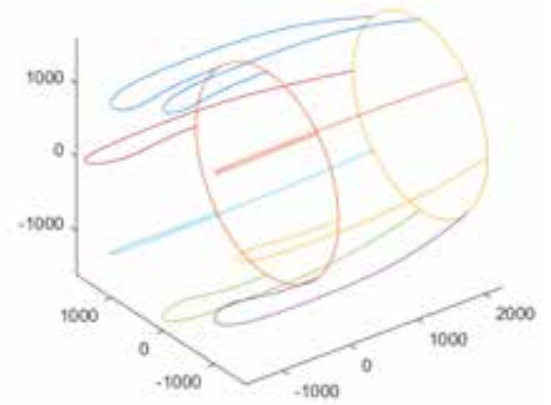

(a) 3D design approach

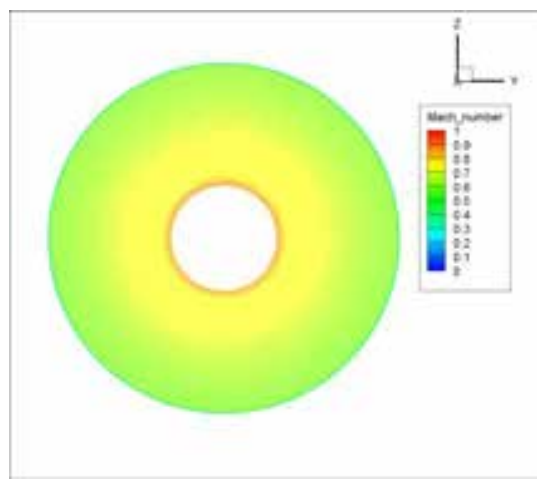

(d) Fan-face Mach number

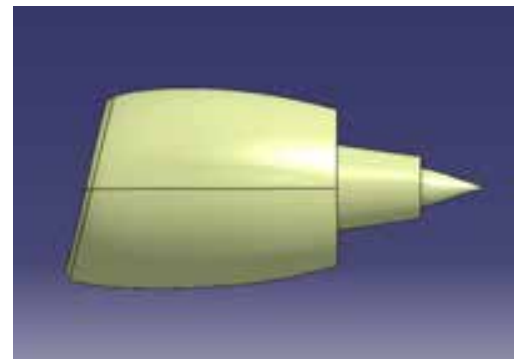

(b) scarfed intake

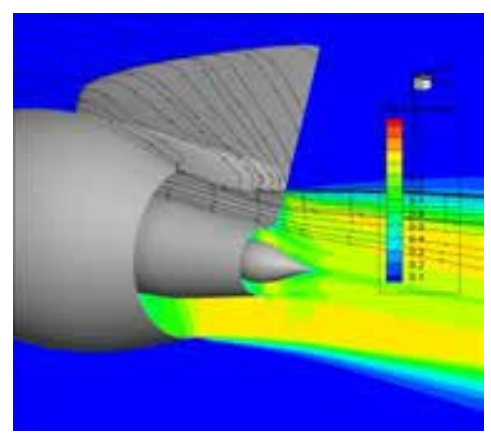

(e) Flow field of static test of nozzle with pylon

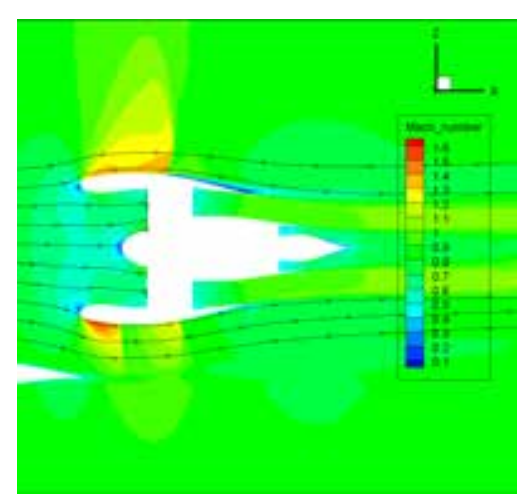

(c) WBE flow field around the nacelle

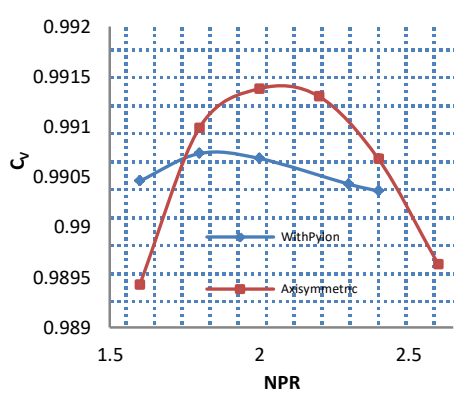

(f) Nozzle characteristics with pylon

Figure 15: 3D design and installation aspects

\section{CONCLUSIONS}

A CFD based nacelle design system has been proposed, which combines an automated CFD analysis system with an adaptive response surface methodology based optimization algorithm. The iCST method was used for complete parametric definition of the nacelle geometry. The approach was applied to the design of the intake, fan cowl, bypass duct and nozzle for a UHBR engine. The parameter trends presented indicate that the optimum predicted by the optimizer is in agreement with physical behaviour of the nacelle. Two design candidates with 23DC and 26.9DC were presented. The response surface was able to predict the cruise drag with $6 \%$ accuracy. The optimized nozzle had a peak $\mathrm{C}_{\mathrm{V}}$ of 0.9914 and corresponding $C_{D}$ of 0.995 . Hence, a holistic nacelle design and optimization system for both intake-fan cowl 
and bypass nozzle design was established. While this study focussed on the design of isolated axisymmetric nacelles, future work would extend the presented approach for the design of three dimensional installed nacelle configurations.

\section{ACKNOWLEDGMENTS}

Financial support from the German Federal Ministry of Economic Affairs and Energy is gratefully acknowledged for funding of the AVACON-project (Grant 20A1702H). The authors would also like to thank Julian Bijewitz and Rasmus Merkler from MTU Aero Engines AG, Patrick Wegener and Fabian Lange from DLR Braunschweig, for their insightful advice and continued support.

\section{NOMENCLATURE}

\begin{tabular}{|c|c|}
\hline AIP & Aerodynamic Interface Plane \\
\hline AR & Aspect Ratio \\
\hline$\beta$ & Boat tail angle \\
\hline BPR & bypass ratio \\
\hline CFD & Computational Fluid Dynamics \\
\hline $\mathrm{C}_{\mathrm{D}}$ & Nozzle discharge coefficient \\
\hline $\mathrm{CP}$ & Charging Plane \\
\hline CR & Contraction Ratio \\
\hline $\mathrm{C}_{\mathrm{v}}$ & Nozzle Velocity coefficient \\
\hline D_fan & Fan Diameter \\
\hline $\mathrm{DC}$ & Drag Counts \\
\hline DC60 & Intake distortion performance metric \\
\hline ESDU & Engineering Sciences Data Unit \\
\hline f_max & Axial Position of Maximum Radius \\
\hline iCST & intuitive Class shape transformation \\
\hline IPR & Intake Pressure Recovery \\
\hline $\mathrm{L}$ & Intake Length \\
\hline LHS & Latin Hypercube Sampling \\
\hline 1_duct & total length of bypass duct and nozzle \\
\hline$\overline{\mathrm{Ma}}$ & Mach number \\
\hline MFCR & Mass flow capture ratio \\
\hline NPR & Nozzle Pressure Ratio \\
\hline R_hi & Highlight Radius of Nacelle \\
\hline R_LE & Leading Edge Radius \\
\hline R_max & Maximum Radius of Nacelle \\
\hline RANS & Reynolds Averaged Navier Stokes \\
\hline RSM & response surface methodology \\
\hline SA & Spalart Allmaras \\
\hline SST & Shear Stress Transportation \\
\hline UHBR & Ultra-High Bypass Ratio \\
\hline $\mathrm{Y}+$ & dimensionless wall distance \\
\hline
\end{tabular}

\section{REFERENCES}

A. Heidebrecht, D. G. (2019). Surrogate model of complex non-linear data for preliminary nacelle design. Aerospace science and technology, 399-411.

B.M. Kulfan, J. B. (2006). Fundamental parametric geometry representations for aircraft component shapes. 11th AIAA/ISSMO Multidisciplinary Analysis and Optimization. Virginia, USA.

Celik, I. B. (2008). Procedure for Estimation and Reporting of Uncertainty Due to Discretization in CFD Applications. Journal of Fluids Engineering, 078001-1-4.

Christie, R. (2016). An autoatated approach to nacelle parameterization using intiutie class shape transformation curves. Journal of Engineering for gas turbines and Power, Vol. 139.

Clemen, C. A. (2012). Systematic Optimisation of a Turbofan Bypass Duct System. ASME, Paper No. GT2012-68276.

Daggett, D. (2002). Ultra Efficient Engine Technology Systems Integration and Environmental Assessment. NASA Glenn Research Center, Paper No. CR-2002-211754.

Dieter Schwamborn, T. G. (2006). The DLR TAU-code: recent applications in research and industry. European Conference on Computational Fluid Dynamics.

F. Zhu, N. Q. (2013). Intuitive class shape function parameterization for airfoils. AIAA Journal, 17-25. 
Goulos, I. (2016). Aerodynamic Design of Separate-Jet Exhausts for Future Civil Aero-engines-Part II: Design Space Exploration,Surrogate Modeling, and Optimization. Journal of Engineering for Gas Turbines and Power, Vol. $138 / 081202-1-12$

Heath, C. M. ( 2015). Aerodynamic Shape Optimization of a Dual-Stream Supersonic Plug Nozzle. SciTech, Reston, VA, AIAA Report No. 2015-1047.

Ioannis Goulos, T. S. (2016). Aerodynamic Design of Separate jet exhausts for future civel Aero-engines part I: parameric geometry defiition and CFD approach. Journal of Engineering for gas turbine and power, 081201-1 081201-14.

Ivo Couckuyt, T. D. (2014). ooDACE Toolbox: A Flexible Object-Oriented Kriging Implementation. Journal of Machine learning research, 3183-3186.

John R. Hooker, A. W. (2013). Over Wing Nacelle Installations for Improved Energy Efficiency. 31st AIAA Applied Aerodynamics Conference. San Diego, USA.

Kulfan, B. (2008). Universal Parametric geometry representation method. Journal of Aircraft, 142-158.

Kulfan, B. M. (2008). Universal Parametric Geometry Representation Method. Journal of Aircraft, Vol. 45.

Matthew Robinson, D. G. (2017). An optimization method for nacelle design. 55th AIAA Aerospace Sciences Meeting. Grapevine, Texas.

Moritz Albert, D. B. (2014). Automatic Design Evaluation of Nacelle Geometry Using 3D-CFD. 15th AIAA/ISSMO Multidisciplinary Analysis and Optimization Conference. Atlanta, GA.

Pavlos K. Zachos, M. F. (2017). Pressure Flow Field and Inlet Flow Distortion Metrics Reconstruction from Velocity Data. AIAA Journal, 2929-2941.

Sebatian Woehler, J. H. (2018). Preliminary aircraft design for a midrange reference aircraft taking advanced technologies into account as part of the AVACON project for entry into service in 2028. Deutscher Luft-und Raumfahrtkongress 2018, (S. DocumentID: 480224).

Stefano Tosin, J. F. (2014). New Approach for Multi-Rotor Mixed-Flow Pump Design and Optimization. 15th Symposium on Turbomachinery Flow Predictions and Optimization, (S. FEDSM2014-21595, V01AT02A007). Illinois, USA.

Suder, K. J. (2013). NASA environmentally responsible aviation project's propulsion technology phase 1 overview and highlights of accomplishments. AIAA Paper No., , 2013-0414.

Tosin, S. (2014). Aerodynamic optimization of turbprop turbine blades using a response surface methodology based algorithm. Proceedings of the ASME 2014 4th Joint US-European Fluids Engineering Division Summer Meeting.

Toubin, H. (2014). Multipoint Aerodynamic High Fidelity Shape Optimization of an Isolated Engine Nacelle. 52nd Aerospace Sciences Meeting. Maryland, USA.

Viall, W. S. (1968). The Engine Inlet on 747. ASME. 